\title{
A Semantic Recommender Engine for Idea Generation Improvement
}

\author{
Maria El Haiba ${ }^{1}$, Lamyaa Elbassiti ${ }^{1} \&$ Rachida Ajhoun ${ }^{1}$ \\ ${ }^{1}$ Smart Systems Laboratory SSL, ENSIAS, Mohammed V University, Rabat, Morocco \\ Correspondence: Maria El Haiba, Smart Systems Laboratory SSL, ENSIAS, Mohammed V University, Rabat, \\ Morocco. Tel: 00212-668-095-448. E-mail: maria.elhaiba@gmail.com
}

Received: July 5, 2018

Accepted: July 19, 2018

Online Published: July 30, 2018

doi:10.5539/cis.v $11 \ln 3$ p 112

URL: https://doi.org/10.5539/cis.v11n3p112

\begin{abstract}
In order to develop the ability to be competitive in considering rapidly growing global market and enormously changing in technology, organizations are looking for up-to-date procedures to respond to all these transformations. Being smart and innovative is actually the most significant pillars of successful organization strategies. In other words, organizations need to encourage learning, manage knowledge and create innovative ideas. A major issue of creative ideation is improving the quality of the ideas generated. In this paper, we propose a semantic recommender engine for idea generation in order to assist organizations to improve their ways of generating new ideas. Through this novel system, innovation actors will be able to consider new perspectives, make new connections, think differently and thus produce new promising ideas. We initially introduce the concept behind a smart organization, explore the idea generation in such organizations and examine the role of recommender systems for managing this stage and identifying breakthrough ideas. Next, we present the context of design, the conceptual architecture of the suggested system and finally expand the workflow of semantic similarity matching of ideas with a focus on the key components of the semantic recommendation engine.
\end{abstract}

Keywords: innovation, smart organization, idea generation, recommender system, architecture, semantic similarity, workflow

\section{Introduction}

Leading an organization to quickly adapt to a dynamic environment is one of the difficult tasks the organizations faces today. The key is on building a smart organization with a strong innovation-driven mindset where the capability to continuously adapt to unpredictable changes is developed. Innovation has actually become vital to most organizations if they want to survive and grow in the long term. Similarly, ideas are vital for organizations because they are the cornerstones of innovation and this in turn is boundless source of competitive advantage (Dorrow et al., 2015). In this respect, we strongly believe that the ability of an organization to grow is reliant on its ability to bring new ideas and to exp loit them efficiently for their long-term benefit. Our works and researches in improving innovation are focused on the first key activity at the Front End of innovation, especially idea generation. The aim is to improve the quality of the submitted ideas and ensure a continuous ideation. Idea generation is a critical, but often overlooked stage at the early stage of innovation projects; defining and understanding this phase is important because it is the starting point of every innovation. In this respect, the generation stage thus needs to be well-managed and requires well-developed mechanisms for gathering ideas with very high quality (El Haiba et al., 2017a). Recommendation systems have so far largely been used in many different fields (i.e. commerce, medicine and so on). Although their relevance, they haven't been yet being used in the innovation management context especially the improvement of the idea generation stage. This paper aims to fill this gap and proposes a novel semantic-based recommender system for idea generation improvement. Th is engine is a part of a larger system we conceived to improve the quality of idea generation and which consists of two main modules: (1) A Semantic Recommender Engine and (2) An Enrichment Engine. This Idea Quality Improvement System aims to drive organizational members to continuously generate and develop innovative ideas. This article introduces the work that is being done to provide the idea generation environment with a semantic recommendation engine.

The remainder of this article is structured as follows: The next section introduces the concept of smart organization while exp loring the need for developing such concept then provides answers to the key challenges it has to face. Section 3 presents the architecture of our proposed system aiming to leverage from reco mmendation systems in the ideation process. Section 4 expand in detail the key component of our semantic idea recommender 
engine and the workflow to be used for semantic similarity matching of ideas. Finally, we draw conclusions and indicate directions for future research in Section 5.

\section{Key Challenges of a Smart Organization}

The main purpose of this section is to present the concept behind smart organization and discuss the improvement of idea generation stage as the first step of the innovation process using recommendation.

\subsection{Smart Organization}

The new economy, deriving from advances in information and communications technologies (ICTs), requires major revisions around how organizations are structured and how they function. Actually, the traditional attitudes and ways of working need to be redefined. However, if organizations want to obtain the benefits from this transformations they will have to put the right policies in place. Hence, the need to develop a dynamic and intelligent organizational model (Brătianu et al., 2006). Being smart and innovative is actually the most significant pillars of successful organization strategies. In a complex, dynamic environment, the positive effects of a smart organization can be more significant compared to the effects in a stable environment (Schafer, 2009).

A Smart Organization is defined as an organization "that acts effectively in the present" and have the competence to "deal effectively with the challenges of the future" (Wiig, 2000). This emerging organizational concept indeed arose from the need for organizations to respond to the increasingly changing business landscape in dynamic, innovative and smarter ways (El Haiba et al., 2017a). Some of the main features of this organizational transformation are the ability to think differently, collaborate, learn, create, distribute and explo it knowledge to promote growth and competitive advantage.

The term "s mart organization" is therefore used by organizations that are internetworked, knowledge-driven, and dynamically adaptive to new organizational forms, learning as well as flexible in their ability to identify and exploit the opportunities offered by the new economy (Filos \& Banahan, 2001). It also means embracing disruption in our organizations to create an environment where innovation is encouraged. Actually, innovation is the key process that can make organizations maintain their market share, improve efficiency and differentiate themselves successfully in their marketplace.

However, managing innovation in such type of organization remains a big challenge and frustrating pursuit despite massive investments in time and money. The critical question here is how can organizations deal with the complex challenges they face under time pressure, and with continuous increase of data?

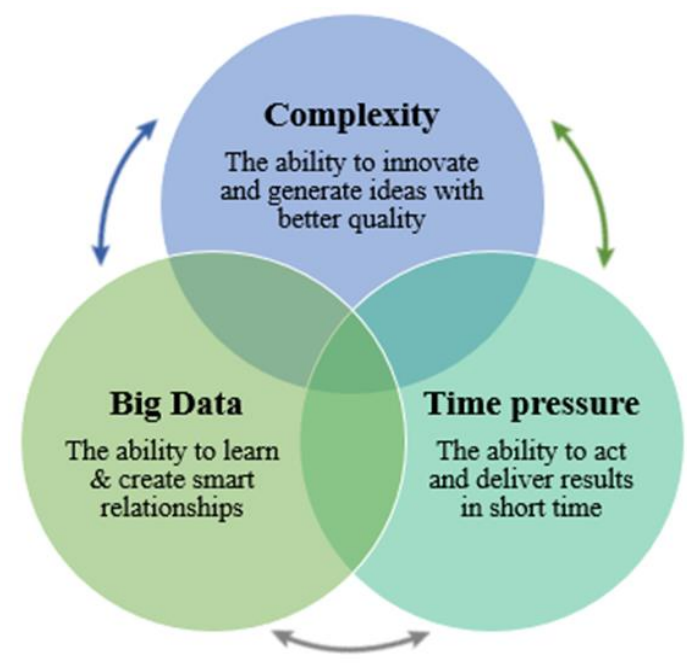

Figure 1. Challenges of Smart Organization

The problem with complex challenges to be faced and the innovation improvement efforts required, in our view, is rooted in the lack of an innovation strategy. Good innovation is no longer random or occasional; but rather structured and well managed. Currently, these challenges are dominantly addressed from a managerial perspective, as advocated by organizational sciences. Such knowledge is indeed necessary for managing an organization, but it is insufficient for bringing about changes in a fully systematic and integrated way. To do that, a smart organization needs to take a constructionaland learning perspective. 
However, a higher degree of "intelligence" would be desirable to reach a perfect state in which learning processes are handled by software that targets and provides just-in-time the learning activities that are needed by the individuals for personal growth (Sicilia \& Lytras, 2005). At the same time, organizations who want to develop and maintain high levels of innovation need a consistent and qualitative flow of generated ideas (Björk \& Magnusson, 2009). Furthermore and in order to effectively manage these ideas, organizations need to adopt smart resources and tools that foster learning activities. To deal with this, a semantic approach can be applied to provide the expected intelligence.

Next subsections will concentrate on presenting idea management systems particularly its idea generation stage, the recommendation as well as the semantics imperatives for enhancing learning and improving the quality of submitted ideas.

\subsection{Idea Management vs Complexity}

Idea management is an integrated part of the innovation process. It is generally represented by systems which principally aids organizations to collect ideas fro $\mathrm{m}$ all emp loyees and evaluate them in order to determine which have the greatest potential to create added value.

In today's competitive context, organizations will increasingly compete based on the speed at which they can find, develop and implement ideas for new products and services. To compete at this level, organizations must productively capitalize on the creativity of all of their members. This paper actually addresses the idea generation stage as one of the most important and critical activities at the front end of innovation.

One of the key success factors to any organization is being able to come up with new ideas to keep operations, products and services up-to-date.The process of capturing those ideas is called ideation or idea generation. All gathered ideas need to be stored efficiently. They are actually the "potential starting point for any innovation venture and by understanding and supporting idea processes in front end innovation companies can strengthen their innovative capability" (Jensen, 2012). Ideas have become the currency of this new economy. They can be sparked by experiences related to a personal observation but also situations that influence the innovator and lead to an act of thought.

Being smart is also means generating ideas but in a structured manner and smarter way. It is no longer simply a question of generating a large number of ideas but capturing those with a high level of quality and which are most likely to become successful innovations. Thus without a mechanis $m$ for managing them, it is difficult to channel innovation activity into the areas it is needed most.

In practice and regardless of the progress in the Idea Management solutions, this initial phase of the innovation process still faces a number of problems. According to Westerski (2013), these problems are typically related to "information overflow and recognizing questionable quality of submissions" with reasonable time and effort allocation. In other words, if organizations want to significantly tap into their employees' initiative taking behavior and specifically their idea submissions, they should address the three common following challenges: idea quantity, idea quality and continued ideation (Deichmann, 2012). To support and facilitate the ideation process, the knowledge about what influences the quality of the ideas created is therefore important. In a previous work, a qualitative approach was developed in response to this increased need to support activities at the generation stage (El Haiba et al., 2017b). The approach is guided and supported at the top by organization structure and processes, its human capital, as well as its knowledge memory. In fact, building a creative organizational structure can motivate everyone to contribute their ideas or opinions and promote a larger nu mber of high quality idea submissions. Also, managing knowledge have a very powerful role in spurring actors to raise their creative output by learning from the performance of their previous ideas. This in fact implies that all parties should be involved at the same time for the benefit of idea generation stage. It is therefore considered appropriate to practically implement this approach and indeed thinking about an ideation process in which employees continuously learn, repeatedly take initiative and produce multiple high-quality ideas.

\subsection{Recommender Systems vs Time Pressure}

All organizations want to come up with the next big idea that catapults their product, their team, or their business to new heights. But where to start and which mechanis ms should they embrace to increase the quality of ideas? It has been shown through the qualitative approach that the generation of new ideas requires well-developed mechanis ms for recognizing and selecting information among a wide range of data (El Haiba et al., 2016). However and under time pressure, there is need to filter, prioritize and efficiently deliver the relevant information. This is where recommender systems have particularly a huge advantage. Previous research study have shown that recommender systems can effectively improve creative ideation output (El Haiba et al., 2016). 
The use of the recommendation within s mart organization will allow it to have a new form that will take into account new components able of en rich ing its potential and improving its effectiveness. We particularly describe the use of a recommender system as the continuous knowledge acquisition which will allow actors continuously learn, generate ideas and collaborate. Some benefits of recommender systems are presented in Figure 2 below.

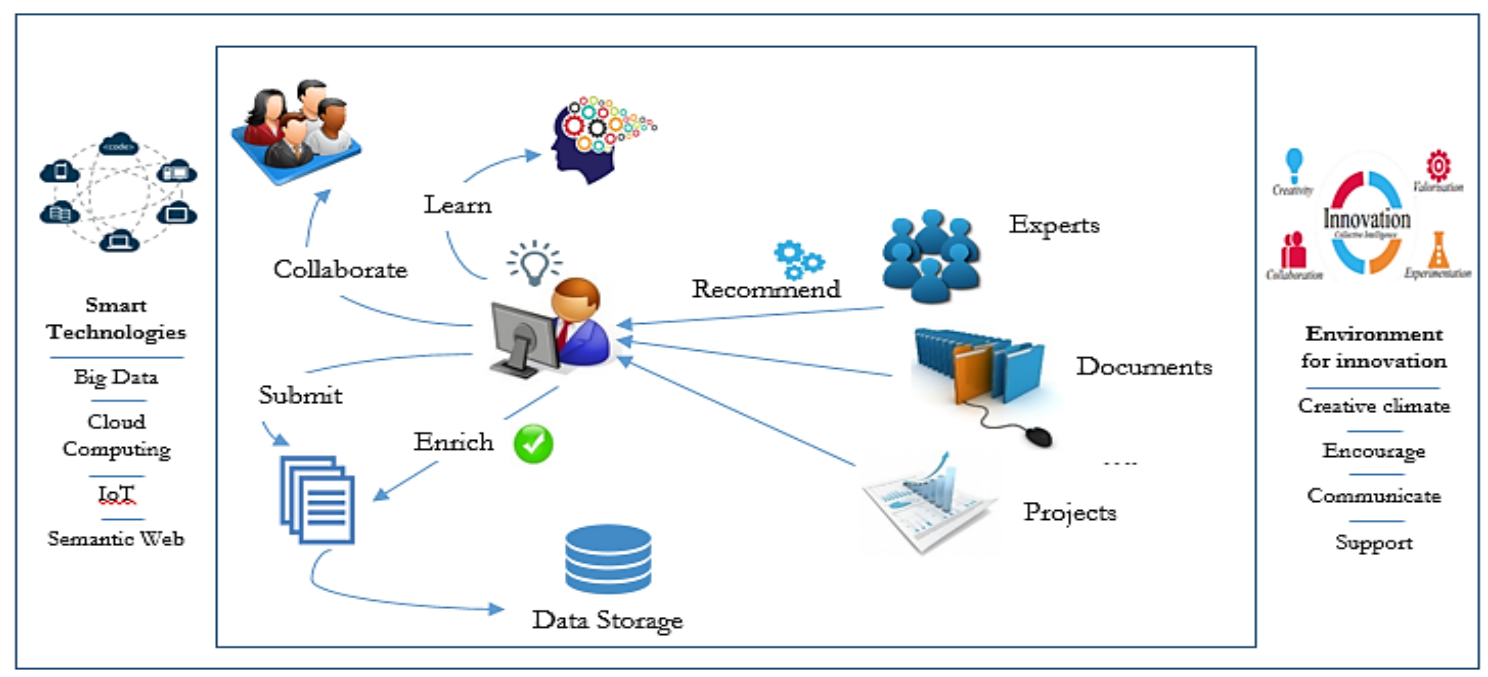

Figure 2. Recommendation in Smart Organization

Recommender systems actually offers relevant items based on the user's interests maintained in its profile. They also help users efficiently overcome the problem of content overload by filtering irrelevant information when users search for desired information (Amini et al., 2011). Relevant knowledge provided by recommended items can lead actors to think differently and thus generate new ideas and learn from prior experiences in order to enrich them and then be stored.

Likewise recommender systems offers opportunities to collaborate by sharing expertise and knowledge in free-flowing and creative ways (from the different knowledge sources: experts, ideas, documents or projects). Experimental research confirms that showing users similar ideas and combining them to build additional ones can be beneficial and inspirational in the idea generation process (Siangliulue et al., 2016). Thus, collaboration during the ideation process will be helpful in repeatedly generating enhanced qualitative ideas over time.

\subsection{Semantics vs Big Data}

The rapidly growing amount of data in every knowledge domain presents challenges to the integration, retrieval and reuse of information relevant to a specific context. To deal with this situation, methods of knowledge representation and analysis play an increasingly major role. Applying Semantic technologies allow in fact the generation of content-rich knowledge models. Big data can in this respect take great advantages from semantics-aware methods, which identify semantic relations among knowledge kept in this massive data in order to extract meaningful information and make better use of it. Actually, semantic technology can make data machine readable, then data can be processed intelligently.

Semantic technologies can be especially beneficial in the improvement of the processes of idea generation as part of the organizational learning. They actually contribute positively to all aspects of organizational cont ent generation, distribution, retrieval and reuse (Sicilia \& Lytras, 2005). Furthermore, such technologies give an opportunity to build implicit connections and smart relationships between knowledge. This indeed led us to talk about semantic learning organization. The "Semantic Learning Organization" is a concept that relates the notion of learning organization to the technological aspect, so that a semantic learning organization can be viewed as a learning organization in which learning activities are improved and mediated through a kind of technology that insures a shared knowledge representation about the context of the organization (Sicilia \& Lytras, 2005). Learning and collaboration activities are greatly facilitated with the use of such integrated semantic tools.

\section{Idea Quality Improvement System}

Our theoretical investigations to prove the role and feasibility of using recommendation systems applications in innovation context were highly promising (El Haiba et al., 2016), but however should be practically 
implemented in order to test the impact of th is new use on both the quality of participations. The main purpose of this section is to briefly present the context of design and then explore in detail the suggested system.

\subsection{Context of Design}

In this work, we investigate ideas that were submitted by innovation actors inside the organization. We are actually interested in ideas as sources of new processes, new products or services, organizational or strategic changes... These ideas may usually serve to prevent anticipated problems, enhance existing structures, or take advantage of specific opportunities. They can be generated in any time. The submission is achieved through a simple input form (idea details submission form) where user fulfills the data re lative to the idea formalization such as: title, keywords, summary etc. The system will also have a provision of user profile creation. Amongst others, users can be informed at generation time about some supplementary criteria for the ideas that the organization currently looks for.

Previous work has shown that standard idea generation techniques are focused on gathering a wide-ranging portfolio of ideas (El Haiba et al., 2017b). This can undoubtedly generate results. But more than that, our objective is to guarantee a continuous flow of new valuable ideas and ensure their continuous development. Consequently, our focus is to propose a new system (ongoing process to generate ideas) that help organizations leap onto a totally different plane. A system which can push the mind to consider new perspectives, make new connections, think diffe rently and thus create new and interesting ideas that will make a difference. Our proposed system architecture consists of two major parts; Semantic Recommendation part and Enrichment part. The result of the recommendation part is a list of similar ideas or projects that can act as an inspiration for further idea improvement and foster collaboration for developing better ideas (El Haiba et al., 2017a). The result of enrichment part is an improved initial idea presented by its generator according to their experiences, expertise and learning process (El Haiba et al., 2017a). In addressing this point we concentrate on individual learning behaviors and especially on experiences that actors accumulate over time based on the results of their creative efforts.

\subsection{Conceptual Architecture}

Successful idea generation is only possible if organizations have a structured idea management system and process in place. Managing ideas falls first and foremost to structuring the way of gathering them. This actually implies having clear guidelines on how employees can submit new ideas and continuously enrich them. The proposed architecture should allow gathering ideas and improving them based on reco mmended items. In other words, provide suggestions to users in order to stimu late their creativity and give them an inspiration for a new submission and/or eventually improve their ones or existing ones.

The figure below presents the conceptual architecture of the proposed system.

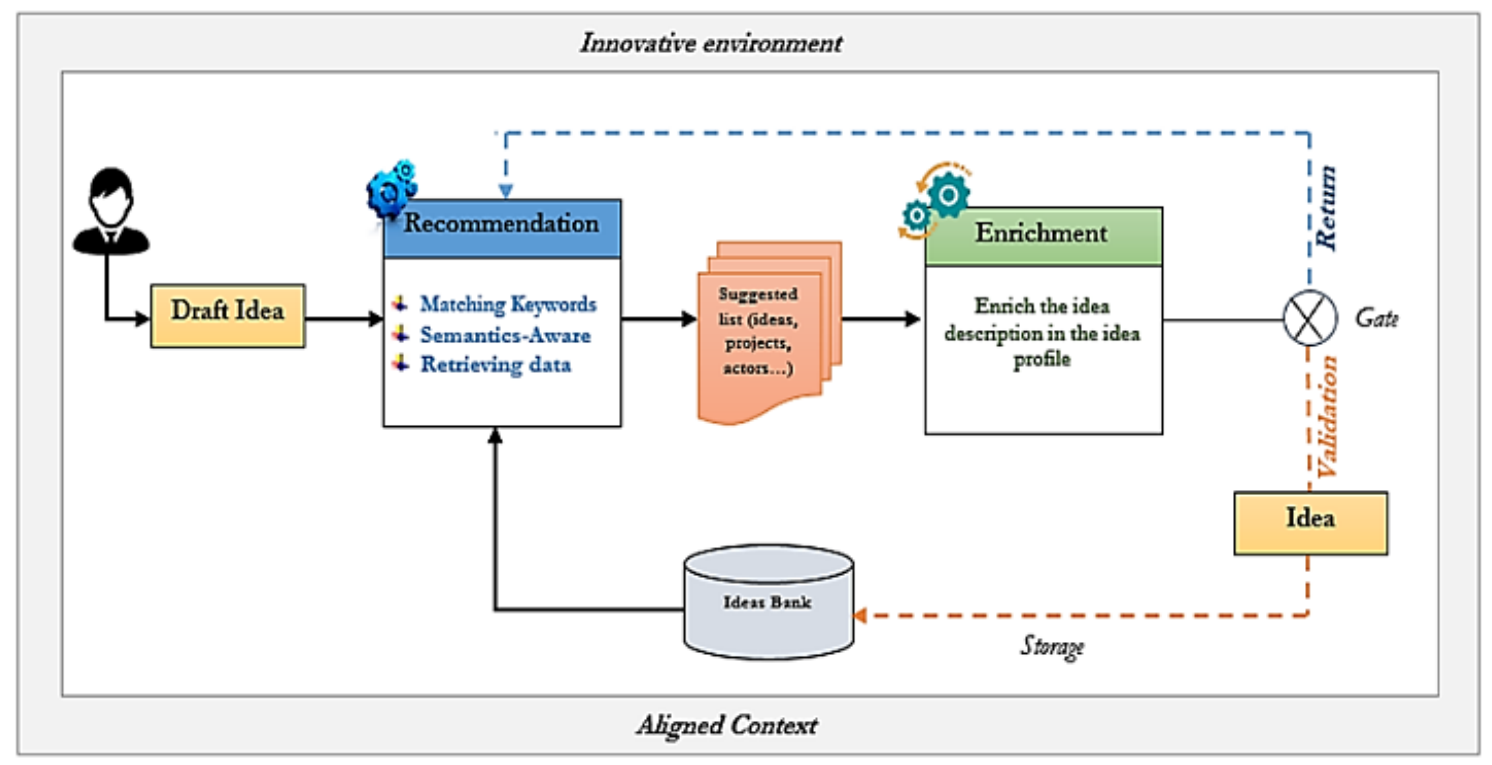

Figure 3. Conceptual System Architecture

An overview of the ideation process is as follows: 
Input data: The set of previously generated or implemented ideas, contextual information related to organization's strategy, goals, and needs. In fact, the ideas that got implemented and furthermo re made very good returns as services or products, can be exposed as success stories. Such practices shall encourage potential innovators to communicate their ideas (Westerski, 2013).

Draft Idea: After the creation of its profile, the user formulates his idea by filling in the corresponding fields in the idea submission form along with attaching images or documents to explain the idea. This filled idea profile highlights the basics behind the new Draft Idea.

Recommender Engine: Once the new draft idea has been submitted, an initial recommending process is made by the system which presents propositions to ideas generators - of the draft idea - in order to help them to enrich their ideas. It support idea refinement by enabling users to examine sets of closely related ideas. The users can view their own ideas and also the repository of the similar ideas submitted. The outcome of this recommendation process is a list of similar or complementary ideas or projects which inspires actors for draft idea improvement and foster collaboration. The collaboration corner - among innovation actors having similar ideas - is actually involved in the matching of ideas and by providing idea and owner details. Recommen dation is achieved by matching of keywords and description from the user's idea with those from ideas of other people while taking into account elements of the context and using semantic similarity measures. Semantic representation of items ideas - is actually intended to reinforce the recommendation by detecting the latent association among the terms that have the same or very similar meaning. The next section explains the components and implemented algorithms of this engine in detail.

Enrichment Engine: The purpose of this part is to propose an automatic enrichment of the draft idea profile. But currently, this process is done manually.

Indeed and after receiving recommendations, the user will try to enrich and re fine his initial idea by taking inspiration from the returned list and/or adapt it according to the current organizational context. Actors can contribute to this by suggesting idea improvements or changes according to their experiences and expertise. This actually involves leaning process - individual and collaborative learning -.

Gate (or decision point): Once the idea is enriched, the user can either receive new recommendations based on the enrichment being made and resume the process or validate his idea. In this case, the idea will be stored in the idea bank. The main aim of this repeated cycle is to activate learning behaviors.

Output data: An idea promising innovation stored in the idea bank. Properly formulated idea outcome data can be used as a motivating factor to generate ideas. So, ideally the stored ideas are reused as inputs for recommendation in order to improve the quality of future ideas.

The outcome of our all work will be a system that is a repository of ideas promising innovation. The system aims actually to ensure that the right ideas end up meeting an organization's relevant innovation needs.

\section{Semantic Recommender Engine for Idea Generation Improvement}

In this section, we present in detail the proposed semantic recommender engine for idea gen eration improvement. The figure 4 below illustrates the overall implementation of the recommendation process particularly the semantic similarity text matching method. The process starts with a repository of textual documents, so-called corpus, which need to be transformed into a vector representation.

Before applying any data analysis technique, a preprocessing activity using Information Retrieval techniques is required. This activity consists in dividing the full text into single words or significant concepts called tokens (tokenization). It also includes process like stop words removal and stemming of word (Arivoli \& Chakravarthy, 2017). It is convenient for taking off common words, such as articles and prepositions (e.g., a, the, at, etc.). The vector itself can be created using different techniques. One of the most practical is the so called "bag of words" method (Vockner et al., 2013). These pre-processing techniques are important since they reduce considerably the number attributes that characterize each document attenuating its dimensionality (Gonçalves et al., 2010). In other words, they are used to reduce the document complexity.

The result of the previous step is an " $n$-dimensional vector space". At this stage, a transformation needs to be applied in order to transfer one vector to another. The standard TF-IDF function can be implemented to assign weights to each term in the document and reflect their relative importance. The purpose of such transformation is to identify semantic relationships between terms, and using them to describe the documents (Vockner et al., 2013). 


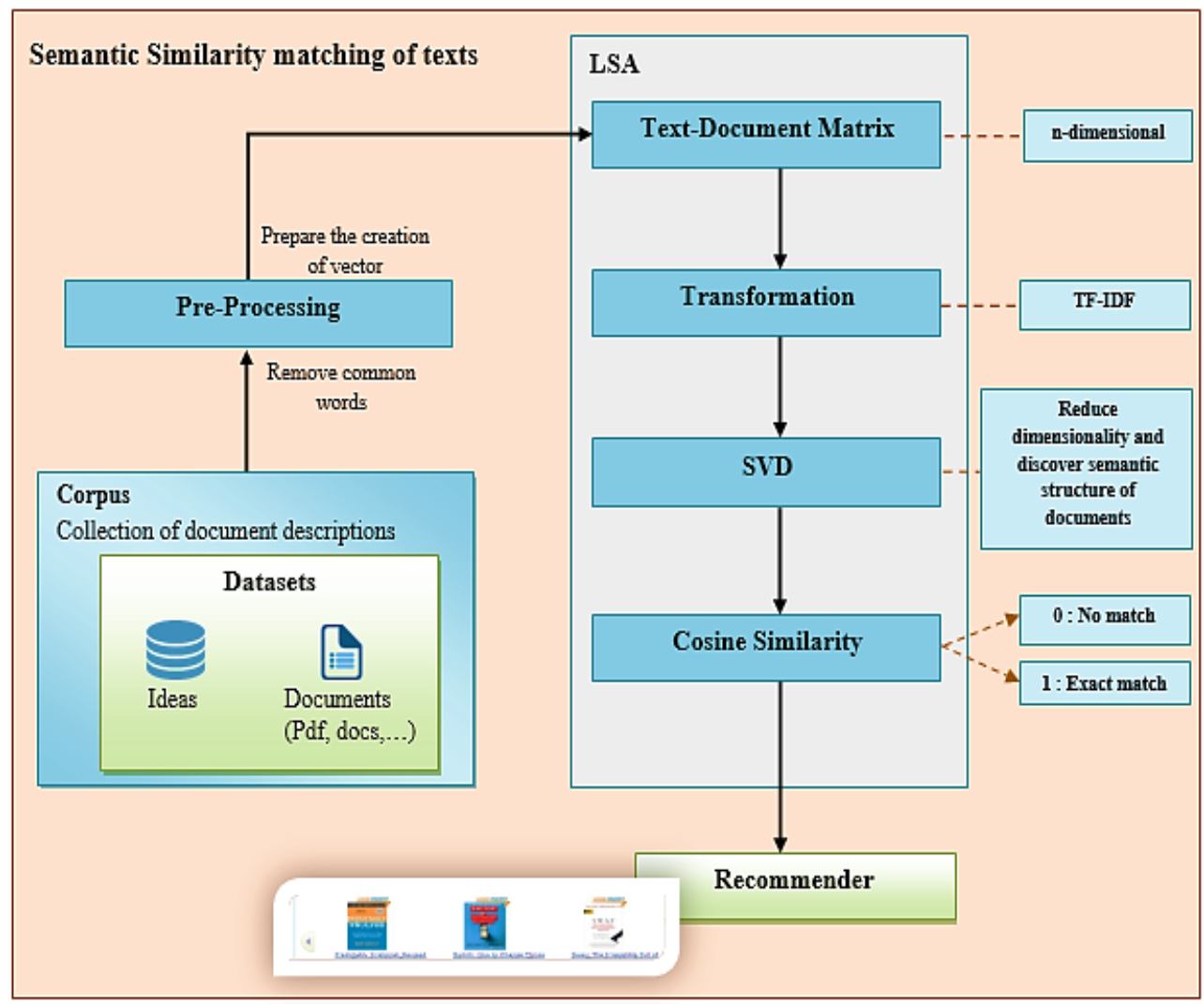

Figure 4. Workflow of semantic similarity matching of ideas

Singular Value Decomposition (SVD) is thereafter applied to reduce the dimensionality of the "n-dimensional vector space" to a lower one. This is essential to discover the semantic structure of the documents. It's actually examine the statistical co-occurrence patterns of words within the corpus. SVD finds a reduced dimensional representation of the matrix that point out the strongest relationships. Therefore, the closely related terms are bundling into the same dimension. Lastly, the similarity between two vectors is calculed with the cosine similarity method. It actually specify if there is an exact/high match (1) or no match (0) at all, with possible degrees in between. At the end, the systemprovides recommendation based of Latent Semantic Analysis method.

These phases will be presented in detail and formalized in the following subsections:

\subsection{Vector Representation of Ideas}

As mentioned previously, the idea had a structured profile with defined fields to fill in the submission form. However, we initially propose to describe the outcome of idea generation stage as an interlinked set of data that can be presented into following:

Table 1. Idea Profile

\begin{tabular}{ll}
\hline Idea Attributes & Description \\
\hline Title & Brief summary of idea \\
Description & Detailed textual description of an idea \\
Keywords & List of words that reflect the main topic of the idea \\
Creation Date & The date when idea was submitted to the system \\
Category & Assignment to some fixed predefined categories \\
Attachments & Rich media like pictures, sketches, videos etc. \\
Actor Information & Idea is interlinked with submitter profile \\
Status & Indicates the position of idea in the organization internal process pipeline \\
\hline
\end{tabular}

The recommendation will be done at the basis of keywords, description and attached document text similarity. 
The content of each item is represented by a set of terms or descriptors, generally the relevant words that appear in a document. A representation that is often used for text documents is the Vector Space Model (VSM) like in the figure below.

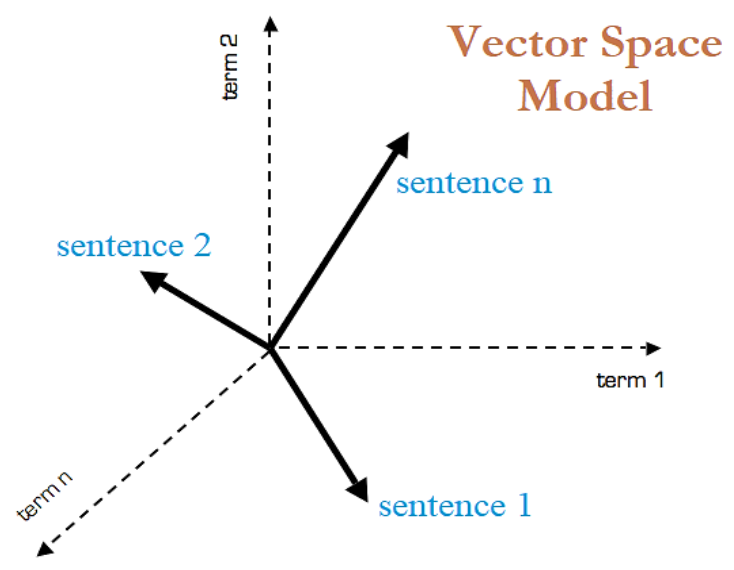

Figure 5. Vector Space Model VSM

Any text can be viewed as a vector in V-dimensional space of a sentence, a query, a document, an entire encyclopedia (Arguello, 2013). This model is an algebraic model which represents text documents as vectors of identifier, such as terms (Zhou et al., 2012). The elements of this vector express the level of important of a word/term and the raw frequency of this word in a document (Huynh et al., 2015).

In our study, ideas will be represented by a vector containing keywords provided by user, extracted keywords from textual description using an algorithm and those extracted from attached document. This in order to enrich the idea vector. However, such representation raise some problems related to synonymy and polysemy. To palliate those drawbacks, Dumais et al. (Dumais et al., 1988) proposed Latent Semantic Analysis (LSA)

\subsection{Semantic Idea Similarity using LSA}

Latent Semantic Analysis or LSA, is a machine learning, statistical and corpus-based method for representing the mean ings of words, sentences and texts. In corpus -based semantic representations, statistical properties of textual structure are exploited to incorporate words in a vectorial space. At this space, words which are similar in terms of meaning tend to be located close to each other.These methods are actually based on the idea that "words with similar meanings tend to occur in similar contexts" (Altszyler et al., 2016). The process of learning words that are related to each other is based on their statistical co-occurrence together in a context (Vockner et al., 2013). Latent Semantic Analysis is one of the most used methods for word meaning representation.

In this study, we describe a keyword extraction technique that uses Latent Semantic Analysis to identify semantically important single topic words or keywords. Using LSA, links between resources are made based on the semantic similarity of their textual content. The main advantage of LSA is that it does not use any manually designed resources, like dictionaries or thesauri. It only depends on large amounts of texts. Forming a corpus, those texts are used to induce knowledge about the meanings of words and documents. LSA, in fact, assumes that "the meaning of a text can be extracted as the sum of the meaning of its words" (Vockner et al., 2013). In contrast to VSM, it extracts concepts instead of words. The basic idea of this method is complete information about the word context. To organize terms and documents into their underlying semantic space, LSA emp loys the singular value decomposition (SVD) algorithm.

LSA includes principally four steps: (1) creation of a term-document matrix out of a collection of texts, (2) application of a transformation with TF-IDF, (3) dimension reduction using Singular Value Decomposition (SVD) and (4) similarity calculation with the cosine similarity measure.

As a first step in using LSA, a sparse input matrix, also called term-document matrix or word-document matrix, is created (Figure 4). Each column of the matrix represents a text passage or sentence in idea (document) under consideration, and each row represents a unique word. Its cell $a_{i j}$ contains occurrence word $w_{i}$ in document $d_{j}$. 


$$
A=\overbrace{\left[\begin{array}{lllr}
a_{11} & a_{12} & \cdots & a_{1 n} \\
a_{21} & a_{22} & \cdots & a_{2 n} \\
\vdots & & & \vdots \\
a_{m 1} & a_{m 2} & \cdots & a_{m n}
\end{array}\right]}^{\text {document }} \begin{aligned}
& \mathbf{m}=\text { rows = terms } \\
& \mathbf{n}=\text { columns }=\text { documents } \\
& \mathbf{a}_{\mathrm{ij}}=\mathrm{w}_{\mathrm{ij}}=\text { term weights }
\end{aligned}
$$

Figure 6. Term-Document matrix A

For transformation or weighting purposes, various methods can be applied. TF-IDF (term frequency-inverse document frequency) and log-entropy are the most common methods (Altszyler et al., 2016). We have used TF-IDF in our study to compute the weight of terms. In particular term frequency specifies the number of times the word occurs in the respective document. Inverse document frequency specifies the relevance of the word respective to the entire document set. The TF-IDF term weight is as follows:

For a term $\mathrm{i}$ in document $\mathrm{j}$ :

$$
\begin{gathered}
w_{i, j}=t f_{i, j} \times \log \left(\frac{N}{d f}\right) \\
t f_{i, j}=\text { number of occurrences of } i \text { in } j \\
d f_{i}=\text { number of documents containing } i \\
N=\text { total number of documents }
\end{gathered}
$$

As the next step of LSA, the SVD decomposes the input matrix A into three matrices:

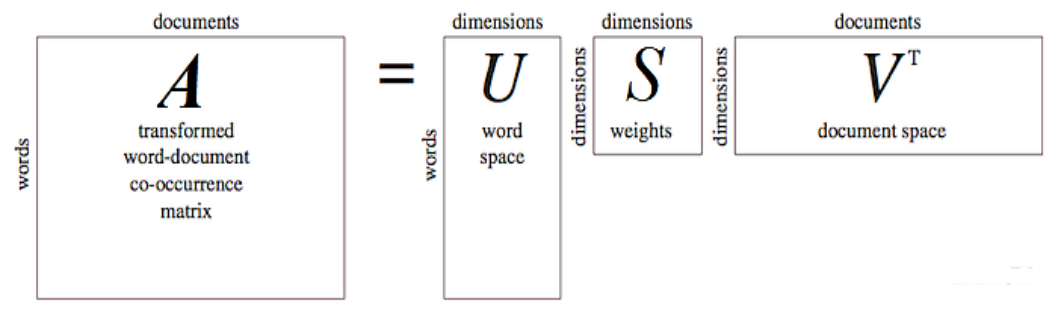

Figure 7. LSA Singular Value Decomposition

Where $A$ is the input matrix, $U$ is the matrix that represents the description of the original rows of the input matrix as a vector of extracted concepts, $\mathrm{S}$ is a diagonal matrix containing scalar singular values sorted in descending order, and $\mathrm{V}$ is an orthonormal matrix that represents the description of the original columns of the input matrix as a vector of the extracted concepts.

Once the vectorial representation of words is obtained, the semantic similarity (S) of two terms is typically calculated using the cosine similarity measure between their respective vectors $\left(\mathrm{v}_{1}, \mathrm{~V}_{2}\right)$ :

$$
S\left(\mathbf{v}_{1}, \mathbf{v}_{2}\right)=\cos \left(\mathbf{v}_{1}, \mathbf{v}_{2}\right)=\frac{\mathbf{v}_{1} \cdot \mathbf{v}_{2}}{\left\|\mathbf{v}_{1}\right\| \cdot\left\|\mathbf{v}_{2}\right\|}
$$

\subsection{Semantic Content-Based Filtering}

Content based filtering algorithms compute the relevance of items or entities based on features ("content") of entities. The most important terms are extracted from the textual description of entities and used for calculating the similarity between the entities (Lommatzsch et al., 2011).

Content-based filtering systems primarly use textual documents as an information source. A standard approach for term parsing selects single words from documents. One of methods that use these terms is the vector space model. As previously defined, it represent documents as vectors in a multi-dimensional space.

The problem of content based filtering techniques is that additional effort is needed for taking into account 
homonyms, synonyms, and anaphors. In this work and in order to overcome those challenges, we consider semantic text matching in combination with recommendations. Semantic text matching, in its simplest form, models the relevance/similarity of a pair of texts considering their relatedness. It captures hidden relationships between terms. We use latent semantic analysis for semantic similarity as shown in figure 4.

The proposed content based recommender system calculates similarity between ideas profile and the other ideas profiles that are submitted by other actors in the organization. Then all similar ideas will be sorted at the basis on the returned similarity value. Finally, the proposed recommender system will recommend top-N similar ideas to the current actor. These recommendations will actually help innovation actors to discover relevant generated ideas, find inspiration for new ideas from a large pool of ideas, improve their own submitted ideas and prevent them from redundant post ideas.

\section{Conclusion}

To ensure long-term competitiveness, organizations need to continuously innovate. Consequently, they also need a continuous stream of ideas as fuel for innovation. The current paper actually explain how organizations can capture new innovative ideas.

The purpose of this paper is to present a new use of recommender system to improve idea generation in the Idea Management Systems. It aims to build a mechanism based on similarity detection to help innovation actors to find content that is relevant in accordance with their needs. More specifically, the paper presents the architecture of a new system designed to express new ideas, store them, and suggest similar ideas to comb ine them and form a better one. Actually, the integration of recommendation techniques as a creativity support to generat ion stage aims to provide people with a seamless experience that enhances their creativity in a natural manner. This in fact spurs idea generation.

As the main result of the presented work, we actually propose the combination of recommender systems with the semantic text matching algorithms in order to enhance the quality of generated ideas in organizations. In this respect, we developed a prototype where structured data (submitted ideas) represent the input for semantic text matching of content using LSA. With this new approach, links between ideas, which were not interlin ked before, can be automatically created. These links are presented using recommendations on the contextual similarity of texts.

Semantic idea recommender system seem to be a promising tool in support of idea generation activities. In terms of future work, we plan to evaluate and experiment our current proposed architecture of the semantic content-based recommender systemin a real application domain for the validation of obtained results.

\section{References}

Altszyler, E., Mariano, S., \& Fernández, S. F. (2016). Comparative study of LSA vs Word2vec embeddings in small corpora: a case study in dreams database. CoRR abs/1610.01520. Retrieved from https://arxiv.org/pdf/1610.01520.pdf

Amini, B., Ibrahim, R., \& Othman, M. (2011). Discovering the impact of knowledge in recommender systems: A comparative study. International Journal of Computer Science and Engineering Survey (IJCSES), 2 (3), 1-14. https://doi.org/10.5121/ijcses.2011.2301

Arguello, J. (2013). Vector Space Model. Information Retrieval, September 25, 2013.

Arivoli, P. V., \& Chakravarthy, T. (2017). Document Classification Using Machine Learning Algorithms - A Review. International Journal of Scientific Engineering and Research (IJSER), 5(2), 48-54.

Björk, J., \& Magnusson, M. (2009). Where do good innovation ideas come from? Exploring the influence of network connectivity on innovation idea quality. Journal of Product Innovation Management, 26(6), 662-670. https://doi.org/10.1111/j.1540-5885.2009.00691.x

Brătianu, C., Vasilache, S., \& Jianu, I. (2006). In search of intelligent organizations. Management \& Marketing, Economic Publishing House, 1(4), (December 2006), 71-82.

Deichmann, D. (2012). Idea Management: Perspectives from Leadership, Learning, and Network Theory. Doctoral dissertation, ERIM, Netherland.

Dorrow, P. F., Davila, G., Varvakis, G., \& Vallejos, R. V. (2015). Generation of ideas, ideation and idea management. Navus, 5(2), 51-59. ISSN 2237-4558. https://doi.org/10.18815/navus.v5i2.248

Dumais, S. T., Furnas, G. W., Landauer, T. K., Deerwester, S., \& Harshman, R. (1988). Using Latent Semantic Analysis to Improve Access to Textual Information. In Proceedings of the SIGCHI Conference on Human 
Factors in Computing Systems, Washington, DC, USA, 15-19 May 1988; 281-285. https://doi.org/10.1145/57167.57214

EL Haiba, M., Elbassiti, L., \& Ajhoun, R. (2016). Can Reco mmender Systems improve Idea Generation Quality in the Front End of Innovation? International Conference on Change, Innovation, Informatics and Disruptive Technology ICCIIDT'16, London- U.K, pp. 37-50.

El Haiba, M., Elbassiti, L., \& Ajhoun, R. (2017a). Smart Organization: Improving Innovation Performance through Recommendation", Proceedings of the $30^{\text {th }}$ IBIMA Conference (International Business Information Management Association), pp. 4557- 4567, Madrid, Spain. ISBN: 978-0- 9860419-9- 0.

El Haiba, M., Elbassiti, L., \& Ajhoun, R. (2017b). Idea Management: Idea Generation Stage with a Qualitative Focus", Journal of Advanced Management Science, 5(4), 271-278. https://doi.org/10.18178/joams.5.4.271-278

Filos, E., \& Banahan, E. (2001). Towards the smart organization: An emerging organizational paradig m and the contribution of the European RTD programs. Journal of Intelligent Manufacturing, 12(2), 101-119. https://doi.org/10.1023/A:1011296325760

Gao, J., \& Zhang, J. (2005). Clustered SVD strategies in latent semantic indexing. InfProcess Manag 2005, 41(5), 1051-1063. https://doi.org/10.1016/j.ipm.2004.10.005

Gonçalves, C. A., Gonçalves, C. T., Camacho, R., \& Eugénio, C. O. (2010). The impact of Pre -Processing on the Classification of MEDLINE Documents. In Ana L. N. Fred, editor, Pattern Recognition in Information Systems, Proceedings of the $10^{\text {th }}$ International Workshop on Pattern Recognition in Information Systems, PRIS 2010, In conjunction with ICEIS 2010, Funchal, Madeira, Portugal, June 2010, 53-61.

Huynh, M. C., Pham, D. T. L., \& Trong, H. D. (2015). Improved Vector Space Model TF/IDF Using Lexical Relations. International Journal of Advanced Computer Research, 5 (21).

Jensen, A. R. V. (2012). A literature review of idea management. Proceedings of NordDesign 2012, The 9th NordDesign Conference, Aalborg University, Center for Industrial Production.

Lommatzsch, A., Till, P., \& Sahin, A. (2011). An architecture for s mart semantic recommender applications. In 11th International Conference on Innovative Internet Community Systems, pages 105-114, Berlin.

Schafer, M. A. (2009). Organizational IQ: Characteristics Common to Smart Organizations and Applicability to the U.S. Military. Master Thesis of Business Administration. The Naval Postgraduate School. Monterey. California. Retrieved from http://www.dtic.mil/dtic/tr/fulltext/u2/a514252.pdf

Siangliulue, P., Joel, C., Steven, P. D., \& Krzyszt, Z. G. (2016). IdeaHound: Improving Large-scale Collaborative Ideation with Crowd-powered Real-time Semantic Modeling. In Proc. of UIST. ACM, pp. 609-624. https://doi.org/10.1145/2984511.2984578

Sicilia, M. Á., \& Lytras, M. D. (2005). The semantic learning organization. The Learning Organization, 12(5), 402-410, https://doi.org/10.1108/09696470510611375

Vockner, B., Andreas, R., \& Manfred, M. (2013). From Geoportals to Geographic Knowledge Portals. ISPRS International Journal ofGeo-Information, 2(2), 256-275. https://doi.org/10.3390/ijgi2020256

Westerski, A. (2013). Semantic Technologies in Idea Management Systems: A Model for Interoperability, Linking and Filtering, (Ph.D. thesis). from http://gi2mo.org/files/papers/thesis2012/westerski_phdthesis_lores.pdf

Wiig, K. (2000). The Intelligent Enterprise and Knowledge Management. Knowledge Research Institute, Inc. prepared for UNESCO's Encyclopedia of Life Support Systems.

Zhou, S. G., Zhang, S. M., \& George, K. (2012). Advanced Data Mining and Applications. 8th International Conference, ADMA 2012 Nanjing, China, December 2012 Proceedings, pp. 323. https://doi.org/10.1007/978-3-642-35527-1

\section{Copyrights}

Copyright for this article is retained by the author(s), with first publication rights granted to the journal.

This is an open-access article distributed under the terms and conditions of the Creative Commons Attribution license (http://creativecommons.org/licenses/by/4.0/). 